\title{
Anti-inflammatory Effect of Ampelocissus Indica (L.) Planch (Chembravalli) in Wistar Rats
}

\section{Nithya V S ( $\nabla$ nithyasarangi3@gmail.com )}

P G Scholar, Department of Dravyaguna vijnanam, Govt. Ayurveda College Thiruvananthapuram https://orcid.org/0000-0002-2064-2620

Indulekha V C

Associate professor , Department of Dravyaguna vijnanam, Govt. Ayurveda College

Thiruvananthapuram

Helen Antony

professor, Department of Biochemistry, University of Kerala, Kariavattom, Thiruvananthapuram

\section{Research}

Keywords: Chembravalli, Ampelocissus indica (L.)Planch, anti inflammatory, Ayurveda, Hortus Malabaricus, Paw edema

Posted Date: September 4th, 2020

DOl: https://doi.org/10.21203/rs.3.rs-54571/v2

License: (c) (i) This work is licensed under a Creative Commons Attribution 4.0 International License.

Read Full License 
The authors have withdrawn this preprint from Research Square 